\title{
Correction to: General Aspects of Exposure Evaluation
}

Christiaan Delmaar, Gerhard Heinemeyer, Matti Jantunen, Klaus Schneider, and Michael Schümann

\section{Correction to:}

Chapter 3 in: G. Heinemeyer et al. (eds.), The Practice of Consumer Exposure Assessment, https://doi.org/10.1007/978-3-319-96148-4_3

The chapter was inadvertently published with an incorrect city "Berlin" in the affiliation of the author Klaus Schneider. This has been updated to reflect the correct city as "Freiburg". 